\title{
Success of patient training in improving proficiency of eyedrop administration among various ophthalmic patient populations
}

This article was published in the following Dove Press journal:

Clinical Ophthalmology

10 August 2016

Number of times this article has been viewed

\section{Alexander Feng \\ John O'Neill \\ Mitchell Holt \\ Catherine Georgiadis \\ Martha MWright \\ Sandra R Montezuma}

Department of Ophthalmology and Visual Neurosciences, University of

Minnesota, Minneapolis, MN, USA

Video abstract

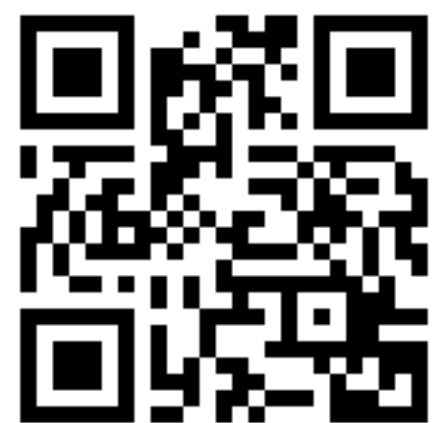

Point your SmartPhone at the code above. If you have a QR code reader the video abstract will appear. Or use: http://youtu.be/-Gruakg4jvE
Correspondence: Sandra R Montezuma Department of Ophthalmology and Visual Neurosciences, University of Minnesota, 420 Delaware St SE, Minneapolis MN

55455-050I, USA

$\mathrm{Tel}+\mathrm{I} 6126254400$

$\mathrm{Fax}+16126263119$

Email smontezu@umn.edu
Purpose: The purpose of this study is to evaluate the success and usefulness of patient education in eyedrop self-administration technique via an educational handout and a short instructional video. Patients and methods: We conducted a prospective study that included 34 patients who were self-administering ophthalmic drops. Of the total patients included, $12 \%$ had used drops for $<12$ months, and $88 \%$ had used drops for $>12$ months. Average age of patients in the study was 67 years, with an age range of 19-91 years. Of the total patients included, $82 \%$ had glaucoma, $6 \%$ had dry eyes, and $12 \%$ did not have a specific diagnosis. Subjects were video recorded and assessed by a trained observer on two occasions: at baseline and after they viewed a demonstrational video and handout. A maximum score of 15 points was awarded based on 15 criteria. A written self-assessment was administered at the end of each study.

Results: Pre- and post-teaching assessment scores improved significantly with education. Patients initially scored an average 2.53 points compared to a post-education score of 6.15 out of 15 points, demonstrating a $2.43(P=0.008)$ factor of improvement. After education, $94 \%$ of patients versus $47 \%$ pre-teaching $(P=0.0001)$ pulled down their lower eyelids. A total of $91 \%$ pre-teaching versus $59 \%$ post-teaching $(P=0.0042)$ patients squeezed one drop into the lower fornix, $74 \%$ pre-teaching versus $26 \%$ post-teaching $(P=0.0002)$ patients released the eyelid and closed the eye for 1 minute, and $56 \%$ pre-teaching versus $3 \%$ post-teaching $(P=0.0001)$ patients applied nasal digital pressure on each eye. We found no significant difference in score changes between those who previously received education and those who had not $(P=0.37)$. A total of $91 \%$ patients responded in a postassessment survey that they now feel more confident of their ability to self-administer eyedrops as their doctor prescribed and that the educational materials were responsible.

Conclusion: Participants demonstrated an immediate and statistically significant improvement in several areas of proper eyedrop self-administration after exposure to a demonstration video and instructional handout.

Keywords: compliance, eye care, patient education, safety, patient satisfaction

\section{Introduction}

Safe and effective technique in the administration of eyedrops is indispensable in the medical management of many acute and chronic ocular diseases contained in our study population, including glaucoma, ocular hypertension, keratoconjunctivitis sicca, postoperative eye care, and others. ${ }^{1-3}$ Improper technique may contribute to excessive medication waste, poorer outcomes, increased costs, decreased efficacy of therapeutic measures, lower patient satisfaction, and may lead to traumatic ocular surface injuries. ${ }^{1-11}$ Previous studies have demonstrated that up to $80 \%$ of patients use an 
incorrect technique when self-administering eye medication, with many lacking formal instruction. In addition, patients have been found to overrate their ability to self-administer eyedrops, which effectively leads to overestimation of compliance rates. ${ }^{12}$ This produces barriers for clinicians to accurately assess the therapeutic response of an ophthalmic medication. Poor proficiency in eyedrop administration may lead to a perceived lack of medication efficacy, when the actual culprit is the lack of contact of the drug with the ocular surface. In addition, improper technique contributes to unnecessary medication waste and increased patient costs, which may amplify patient frustration and lead to worse patient compliance. ${ }^{4}$ Many factors contribute to patient nonadherence, including: visual acuity, eyedrop bottle shape and size, force required to squeeze one drop, angle of the dropper during administration, number of medications patients are currently taking, and the complexity of the administration schedules. ${ }^{13}$ It is arguable that patient education on proper eyedrop administration may be one of the simplest and most cost-effective ways to increase both compliance and clinical outcomes. ${ }^{7,12,14,15}$ Special mention should be made to McVeigh and Vakros, whose team conducted a similar study and found that patients improved in nine out of eleven categories regarding drop administration and compliance after use of an educational handout similar to the results of our study. ${ }^{14}$ Given the crucial function that topical ophthalmic medications have in the treatment of both acute and chronic ocular disease, our study aimed to evaluate the utility and impact of patient education on proper eyedrop self-administration technique in the form of an instructional video, illustrated educational handout, and pre- and post-teaching surveys.

\section{Patients and methods}

This prospective study was approved by the Institutional Review Board of the University of Minnesota. Informed consent was obtained from all participants. Inclusion criteria for study patients included: age $>18$ years, use of one or more ocular medications in one or both eyes, self-administration of ocular medications, and the ability to complete a self-assessment survey. Exclusion criteria for study patients included: patients not on any regularly prescribed ocular medication, age $<18$ years, persons other than patient administering eyedrops, and allergy or sensitivity to fluorescein. The total number of participants was $34(n=34)$ with an average age of 67 (range 19-91) years, and all with visual acuity of $>=20 / 60$ in at least one eye. Recruitment was completed through comprehensive and subspecialty ophthalmology clinics at the University of Minnesota.
Subjects were video-recorded self-administering artificial tears from a sterile bottle on two occasions: at baseline and 5 minutes after the educational tools of an instructional video on proper eyedrop administration technique and an illustrated educational handout were employed. Each recording took place on the same day during the same visit. The patients completed a written self-assessment questionnaire before and after the educational materials were presented (Table 1). A trained observer completed a pre- and post-teaching checklist detailed below and utilized a fluorescein strip to examine the ocular surface following self-administration of the initial eyedrop by the participants (Table 2). Video recording scores were determined by the same observer who was aware of when training materials had been released for each participant.

\section{Statistical analysis}

Stratification of the efficacy of patient educational tools in eyedrop self-administration improvement was determined utilizing the chi-square test (Table 3). Overall measurement of improvement in scores was determined utilizing the Pearson's $t$-test. All $P$-values were considered statistically significant when the values were $<0.05$. The significant variables were modeled alone and in combination against the dependent variable. Data management was performed using Microsoft Excel 2010 (Excel version 14, Microsoft Corporation, Redmond, WA, USA).

Table I Patient self-assessment questionnaire to be completed prior to demonstrational video and handout

I. Visual acuity? (Right eye/left eye)

2. Do you require assistance to complete this form? (Yes/no)

3. Age?

4. Ocular history/medical history?

5. Do you manage and administer your own eyedrops? (Yes/no)

6. What is your handedness? (Right/left)

7. Has anyone ever taught you the proper way to administer your eyedrops? (Yes/no)

8. How long have you been using eyedrops of any kind? (0-6 months, 6-12 months, I year or longer)

9. How many different eyedrops do you use? (I-2, 3, 4 or more, I don't know)

10. How confident are you in your ability to administer your eyedrops as your doctor prescribed? (Very confident, confident, not very confident, not confident at all)

II. How often are you worried you have missed your eye(s) when administering your drops? (Never, sometimes, often, always)

12. Do you ever worry you have touched your eyedropper bottle to your eye(s) while administering your drops? (Never, sometimes, often, always)

13. Do you think that tutoring materials such as an instructional pamphlet and an educational video would help you in properly administering your eyedrops? (Yes/no) 
Table 2 Pre- and post-teaching observer assessment checklist

I. Shake medication? (+I if shakes, $-\mathrm{I}$ if not)

2. Wash hands? (+I if washes hands, $-I$ if not)

3. Touch tip of dropper to anything? ( $-I$ if touches, $+I$ if not)

4. Tilt head backward? (+I if tilts head, $-I$ if not)

5. Look up when administering eyedrops? (+I if looks up, $-\mathrm{I}$ if not)

6. Pull down lower eyelid to form a pocket? (+I if pulls down, $-I$ if not)

7. Squeeze one drop into fornix? ( + I if one drop, $-I$ if $>$ I drop)

8. Avoids touching dropper to eye? ( $+I$ if avoids, $-I$ if not)

9. Release and close eye for I minute? (+I if releases, $-I$ if not)

10. Apply pressure at punctum for I minute? (+I if applies pressure, -1 if not)

II. Wipe away excess tears with tissue? (+I if wipes away, - I if not)

12. Replace bottle cap without touching? ( - I if touches, + I if not)

13. Attempts ( - I for attempts beyond I) - Right eye?

14. Attempts ( $-I$ for attempts beyond I) - Left eye?

15. Corneal abrasions after administration? $(1=$ no, $-1=$ yes $)$

Note: Patients were given a score of I point for each task performed successfully and $-I$ if unsuccessful.

\section{Results}

In total, 34 patients who self-administered eyedrops were evaluated and surveyed, with an average age of 67 years and age range of 19-91 years. We found that $12 \%$ of patients had been utilizing eyedrops for 0-12 months, while $88 \%$ had been utilizing eyedrops for $>1$ year. Each patient had at least $20 / 60$ or better visual acuity in at least one eye. We did not observe any ocular surface injuries resulting from eyedrop administration.

A pre-teaching survey found that $47 \%$ of patients self-reported that they had never been taught proper eyedrop administration (Table 4). When queried on their ability to properly administer eyedrops, $88 \%$ of participants reported that they were very confident or confident and $12 \%$ stated they were not very confident. When prompted how often they worry about missing the eye during eyedrop self-administration, $9 \%$ of participants reported they always worry compared to $3 \%$ who often worry, $56 \%$ who sometimes worry, and $32 \%$ who never worry. When asked how often they worry about touching the dropper to the surface of their eyes, $59 \%$ of patients reported they never worry compared to $35 \%$ who sometimes worry, $6 \%$ who often worry, and $0 \%$ who always worry.

Post-teaching surveys revealed that $91 \%$ of patients now feel more confident of their ability to administer eyedrops prescribed by their doctor and that tutoring materials helped them to do so (Table 5).

Based on the Pre- and Post-teaching Observer Assessment Checklist, on average, when compared to pre-teaching assessment values as rated by the trained clinical observer, the post-teaching individual scores of participants improved by a factor of 2.43 compared to their baseline $(P=0.008)$ after the institution of educational materials (Figure 1). We found that the eyedrop self-administration technique of patients improved significantly in four specific areas (Table 3). First, when participants were stratified according to those who pulled their lower eyelid, 94\% ( $\mathrm{N}=32)$ of post-teaching patients performed the movement compared to $47 \%$ (N=16) of pre-teaching patients ( $P=0.0001)$. Second, when stratified according to those who squeeze one drop into the pocket formed by pulling the lower eyelid, $91 \%(\mathrm{~N}=31)$ of post-teaching patients performed the movement compared to $59 \%(\mathrm{~N}=20)$ of pre-teaching patients $(P=0.0042)$. Third, when stratified according to those who released the eyelid after drop administration and subsequently closed eyelid for 1 minute, $74 \%(\mathrm{~N}=24)$ of post-teaching patients performed the movement compared to $26 \%(\mathrm{~N}=9)$ of pre-teaching patients $(P=0.0002)$. Finally, when stratified according to those who applied pressure over the punctum for lacrimal

Table 3 Pre- and post-teaching observer to patient assessment questionnaire results

\begin{tabular}{|c|c|c|c|}
\hline & Pre-education (\%) & Post-education (\%) & P-value \\
\hline Shake medication & $2 \mathrm{I}(\mathrm{N}=7)$ & $29(\mathrm{~N}=10)$ & 0.58 \\
\hline Wash hands & $3(N=2)$ & $9(\mathrm{~N}=3)$ & 0.61 \\
\hline Touch tip of dropper to anything & $32(\mathrm{~N}=\mathrm{II})$ & $29(\mathrm{~N}=10)$ & I \\
\hline Tilt head backward & $91(\mathrm{~N}=3 \mathrm{I})$ & $94(\mathrm{~N}=32)$ & I \\
\hline Look up when administering eyedrops & $88(N=30)$ & $97(\mathrm{~N}=33)$ & 0.36 \\
\hline Pull down lower eyelid to form pocket & $47(\mathrm{~N}=16)$ & $94(N=32)$ & 0.0001 \\
\hline Squeeze one drop into pocket & $59(\mathrm{~N}=20)$ & $91(\mathrm{~N}=3 \mathrm{I})$ & 0.0042 \\
\hline Avoid touching dropper to surface of eye & $38(\mathrm{~N}=13)$ & $29(\mathrm{~N}=10)$ & 0.61 \\
\hline Release and close eye for I minute & $26(N=9)$ & $74(N=25)$ & 0.0002 \\
\hline Apply pressure at punctum for I minute & $3(N=1)$ & $56(N=19)$ & 0.0001 \\
\hline Wipe away excess tears with tissue & $76(\mathrm{~N}=26)$ & $79(\mathrm{~N}=27)$ & I \\
\hline Replace bottle cap without touching surface & $68(\mathrm{~N}=23)$ & $79(\mathrm{~N}=27)$ & $0.4 I$ \\
\hline Do not require more than one attempt to administer one drop & $29(\mathrm{~N}=10)$ & $24(\mathrm{~N}=8)$ & 0.78 \\
\hline
\end{tabular}


Table 4 Results of pre- and post-teaching observer's criteria used to grade proper eyedrop use

\begin{tabular}{lll}
\hline $\begin{array}{l}\text { Ever been taught proper } \\
\text { drop administration? }\end{array}$ & Yes 53\% (N=I8) & No 47\% (N=16) \\
$\begin{array}{l}\text { How confident are you in } \\
\text { your ability to administer }\end{array}$ & Very confident & $44 \%(\mathrm{~N}=15)$ \\
drops as prescribed? & Nonfident & $44 \%(\mathrm{~N}=15)$ \\
How often are you worried & Never & $12 \%(\mathrm{~N}=4)$ \\
you have missed your eye(s) & Sometimes & $32 \%(\mathrm{~N}=1 \mathrm{I})$ \\
when administering your & Often & $56 \%(\mathrm{~N}=19)$ \\
drops? & Always & $3 \%(\mathrm{~N}=1)$ \\
$\begin{array}{l}\text { Do you ever worry you have } \\
\text { touched your eyedropper }\end{array}$ & Never & $9 \%(\mathrm{~N}=3)$ \\
bottle to your eye(s) while & Sometimes & $59 \%(\mathrm{~N}=20)$ \\
administering your drops? & Always & $35 \%(\mathrm{~N}=12)$ \\
$\begin{array}{l}\text { Do you think that tutoring } \\
\text { materials such as an }\end{array}$ & Yes $65 \%(\mathrm{~N}=22)$ & $0 \%(\mathrm{~N}=0)$ \\
instructional pamphlet & & \\
and an educational video & & \\
would help you in properly & & \\
administering your eyedrops? & & \\
\hline
\end{tabular}

occlusion, $56 \%(\mathrm{~N}=19)$ of post-teaching patients performed the movement compared to $3 \%(\mathrm{~N}=1)$ of pre-teaching patients. Curiously, when stratified according to those who have been formally taught proper eyedrop instillation, we did not find any statistical difference in score changes between the trained and the untrained groups $(P=0.37)$ (Figure 2).

Areas that did not show significant improvement include: shaking the medication prior to instillation $(P=0.40)$, in which ten post-education versus seven pre-education patients performed the movement; washing hands $(P=0.30)$, in which three patients post-education versus one pre-education patient performed the movement; touching the dropper to anything $(P=0.79)$, in which ten post-education versus eleven preeducation patients performed the movement; tilting the head backward $(P=0.64)$, in which 32 post-education compared to 31 pre-education patients performed the movement; looking up when administering drops $(P=0.16)$, in which 33 posteducation versus 30 pre-education patients performed the movement; touch dropper to the surface of the eye $(P=0.44)$, in which ten post-education versus 13 pre-education patients

Table 5 Post-teaching patient self-assessment questionnaire results

\begin{tabular}{|c|c|c|}
\hline $\begin{array}{l}\text { Do you think that the tutoring } \\
\text { materials will help you in properly } \\
\text { administering your eyedrops? }\end{array}$ & Yes $91 \%(\mathrm{~N}=31)$ & No $9 \%(\mathrm{~N}=3)$ \\
\hline $\begin{array}{l}\text { Do you now feel more confident in } \\
\text { your ability to properly administer your } \\
\text { eyedrops as your doctor prescribed? }\end{array}$ & Yes $91 \%(\mathrm{~N}=3 \mathrm{I})$ & No $9 \%(\mathrm{~N}=3)$ \\
\hline
\end{tabular}

performed the movement; replacing the bottle cap without touching anything $(P=0.27)$, in which 27 post-education versus 23 pre-education patients performed the movement; and making multiple attempts in the right $(P=0.32)$ and left $(P=0.42)$ eyes, in which four post-education versus seven pre-education patients initiated multiple attempts in the right eye and six post-education versus nine pre-education patients initiated multiple attempts in the left eye.

\section{Discussion}

Clinical outcomes and patient compliance have a direct correlation with the ability to effectively and definitively administer ophthalmic medications. Unsurprisingly, patients who are unable to self-administer topical eyedrops may generally trend toward poorer outcomes. Patients may then become frustrated, leading to lower compliance. ${ }^{4}$ As patients tend to overestimate their capacity to deliver eyedrops as well as their compliance rates, it may therefore be difficult for physicians to discern if the perceived lack of drug efficacy is due to treatment failure or the technique patients utilize to deliver such medications. ${ }^{5-7}$

Several studies have noted the importance of educating patients on proper eyedrop administration, but few have actually studied the effects of education on patient technique. ${ }^{12}$ One study by Ritch et al measured the effects a new training technique based on proprioception, and showed significant improvement in patient instillation technique. ${ }^{4}$ Another study by McVeigh and Vakros also utilized a pre- and posttest method in assessing whether an educational handout (Eye Drop Chart), improved patient technique and compliance. ${ }^{14}$ The group found that patients improved in four out of the eleven assessed categories, including punctal occlusion techniques, stating whether they always wash their hands, and shaking the bottle. Like their study, we found patients had improved significantly in punctal occlusion techniques and duration of duct occlusion, but unlike their study, we did not find statistical significance in hygiene-related behavior, including washing hands. This may be due to how data was collected. We physically observed in a clinic setting if patients washed or did not wash their hands, while McVeigh and Vakros's team asked whether patients did so; it may be that compliance rates would have been higher had we asked patients instead of observing whether they performed the physical activity itself. We also did find a significant difference in whether patients shook their medications or not compared to the study by McVeigh and Vakros, although this may be more due to our small sample size. Our study aimed to specifically measure the direct effects educational materials had on patient 


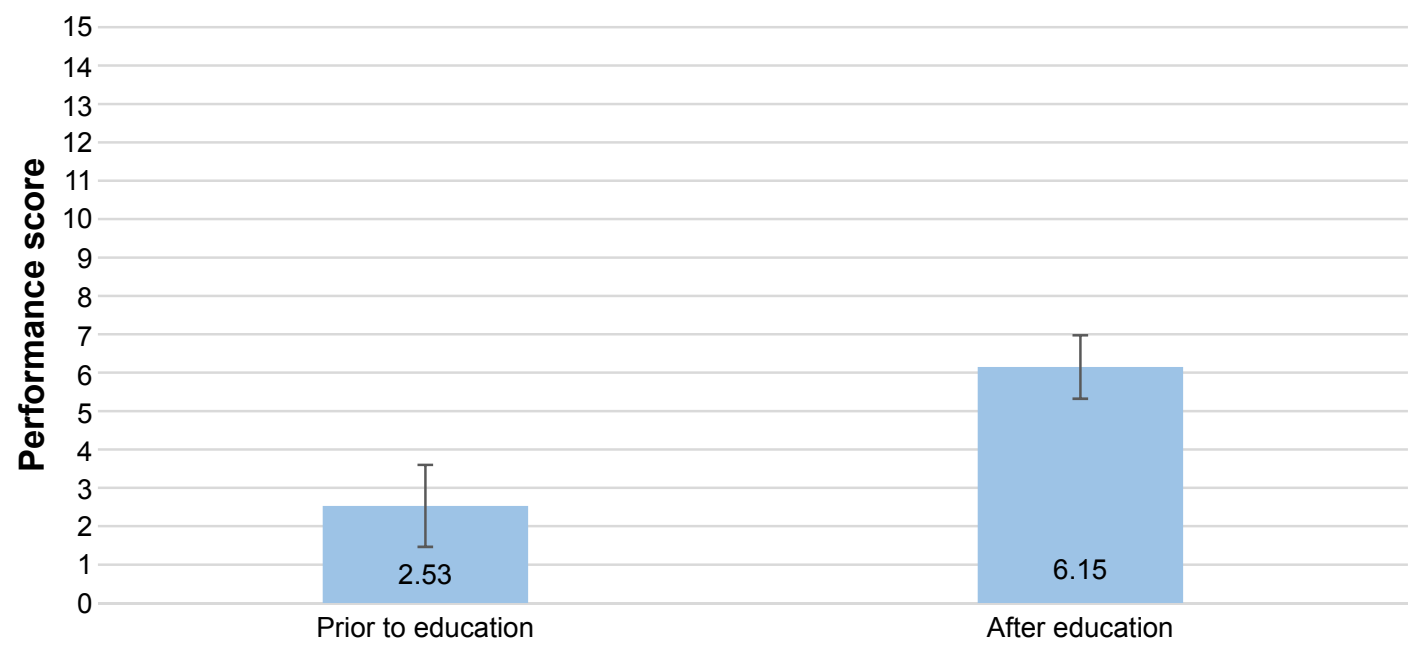

Figure I Total performance scores of eyedrop self-administration pre- and postexposure to educational tools.

Notes: $\mathrm{N}=34$ for both groups; mean $=2.53$ for group prior to education, 6.15 for group after education; standard deviation $=6.22$ for group prior to education, 4.82 for group after education; standard error of the mean $=1.07$ for group prior to education, 0.83 for group after education. Graph demonstrates that, prior to implementation of educational materials, patients scored significantly lower than after they had been taught how to properly administer eyedrops. $P=0.008$.

eyedrop self-administration technique. In addition, our survey questionnaire included several attributes that other studies have noted to be important with regard to proper technique, including whether patients pulled down their lower eyelid when administering a drop, avoided touching the dropper to the surface of the eye, tilting the head backward, looking up when administering drops, and wiping away excess tears. ${ }^{2,46,12,14,16,17}$ We also video-recorded each patient during every eyedrop session to ensure accurate assessment of patient performance.

Our study demonstrated that patient assessment scores by a trained observer significantly improved after a short educational briefing on proper eyedrop administration (Figure 1). Additionally, 91\% ( $\mathrm{N}=31)$ of patients reported in the post-teaching assessment survey that the educational materials aided them by giving them more confidence in their ability to properly administer eyedrops (Table 5). This favorable patient outcome has the potential for significant benefit in many areas of ophthalmology, including improving cost-effectiveness, decreasing health care costs, limiting medication waste, improving clinical outcomes, patient satisfaction, and compliance. However, several limitations of this study exist, which include a relatively small number of patients, patient recall bias, pressure to perform under observation/video, and lack of long-term outcomes. As most of our patients were referred through the glaucoma clinic, other factors unable to be accounted for in the study include comorbid diseases affecting movement, such as arthritis and Parkinson's, which can affect patient performance.

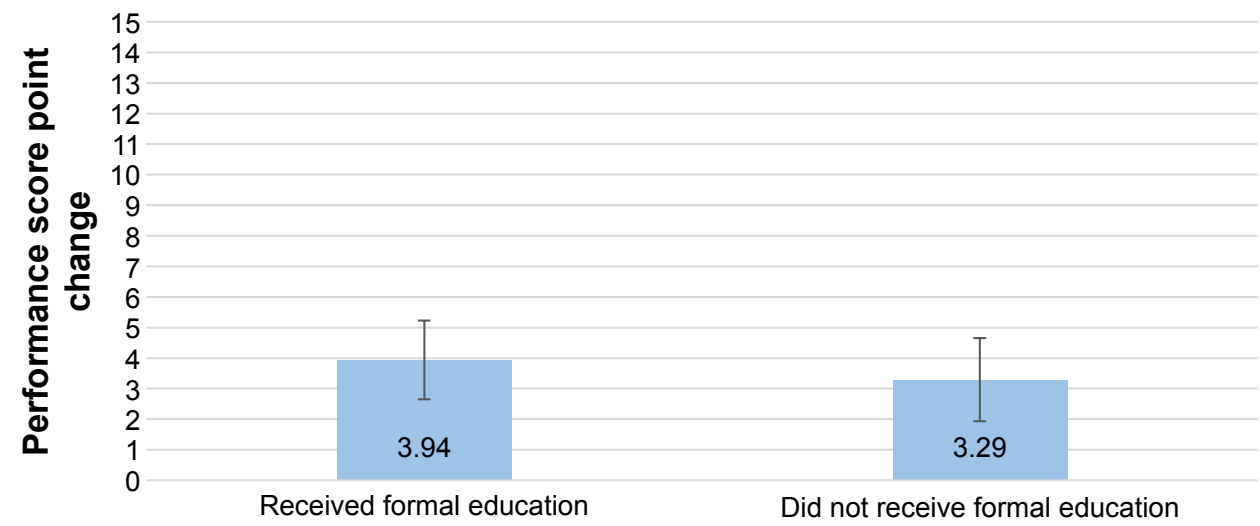

Figure 2 Performance score changes of eyedrop self-administration pre- and postexposure to educational tools broken down by previous educational experience. Notes: $\mathrm{N}=17$ for both groups. For group with participants who had received previous formal education on proper eyedrop self-administration, mean $=3.94$, standard deviation $=5.3 \mathrm{I}$, and standard error of the mean $=1.29$. For group with participants who had not received previous formal education, mean $=3.29$, standard deviation $=5.60$, and standard error $=1.36$. Graph demonstrates that post-education score improvement was not significantly different based on history of previous formal training of proper eyedrop administration. $P=0.37$. 
Areas of assessment that did not show significant improvement should be mentioned, most significant of which include assessments regarding hygiene, including not washing hands, touching the dropper tip to both the eye and other environmental objects, replacing the bottle cap and touching the dropper tip, and making multiple attempts per eye. Each of these have the potential to cause infection and the combination of all may compound that risk. Of specific note, we found that only 3\% $(\mathrm{N}=1)$ washed their hands prior to patient education, and only $9 \%(\mathrm{~N}=3)$ after patient education washed their hands. This may be an aspect related to patient expectations and pressure to perform on the technique of eyedrop administration (tilting head back, closing eye for 1 minute, pulling the lower eyelid to form a pocket, which did show significant improvement) as opposed to focusing on tasks that are perceived to be easier and requiring only common sense. However, the lack of improvement in these areas may be indicative of how patients respond to tasks they are already familiar with, so targeting patients early on when they first begin using eyedrops may be more effective than attempting to educate those who have been utilizing them for a much longer period. However, since changes in scores did not depend on whether patients had received previous formal training or not $(P=0.37)$, physicians may be inclined to provide formal education on proper administration, even though the patient had already been trained in the past. As such, results of the study may be biased toward patients with existing long-term chronic conditions, as $>88 \%$ of patients had been using eyedrops for $>12$ months. Further studies are needed to evaluate for lasting effects following patient education, the clinical benefits and cost-effectiveness of improved eyedrop performance, and to evaluate patient performance with educational tools and eyedrop aids. The educational tools used in this study are inexpensive, effective, and easily distributed.

Our study concluded, in agreement with others, that patients tend to overrate their ability to properly instill eyedrops. We found that more than $88 \%$ of the patients said that they were either confident or very confident in their ability to self-administer eyedrops despite relatively low performance scores on the assessment checklist (Table 1). Poor technique contributes to many negative outcomes, including increased waste and costs given the high price tag for numerous ophthalmic medications. For example, based on Medicaid's National Average Drug Acquisition Cost report for March 2016, some commonly prescribed topical antibiotics, glaucoma and anti-inflammatory medications, such as nepafenac ophthalmic, may cost up to $\$ 362$ per $\mathrm{mL}$, making wasting drops by missing the eye during administration very expensive. ${ }^{18}$

\section{Conclusion}

Overall, patients demonstrated an immediate significant improvement after exposure to an instructional video on proper eyedrop administration technique and an illustrated educational handout. Regardless of experience, prior education, or confidence, the majority of patients reported that tutoring materials have helped them in properly administering eyedrops (Table 2).

Clinicians must consider the role of over-the-counter ophthalmic medications in patients without proper drop administration technique. These patients are likely more susceptible to improper use of medication, medication waste, dropper contamination, and ocular surface injury. ${ }^{19}$ We propose that clinicians consider including formal yet inexpensive patient education tools, such as demonstrational videos and instructional handouts, in workflow to promote patient comprehension, compliance, and satisfaction.

\section{Acknowledgment}

This research was supported by funds from the Minnesota Lions Vision Foundation.

\section{Disclosure}

The authors report no conflicts of interest in this work.

\section{References}

1. Gaynes BI, Singa RM, Schaab G, Sorokin Y. Impact of administration angle on the cost of artificial tear solutions: does bottle positioning minimize wastage? J Ocul Pharmacol Ther. 2007;23:196-201.

2. Tatham AJ, Sarodia U, Gatrad F, Awan A. Eye drop instillation technique in patients with glaucoma. Eye (Lond). 2013;27(11):1293-1298.

3. Olthoff CM, Schouten JS, van de Borne BW, Webers CA. Noncompliance with ocular hypotensive treatment in patients with glaucoma or ocular hypertension: an evidence-based review. Ophthalmology. 2005;112:953-961.

4. Ritch R, Jamal KN, Gurses-Ozden R, Liebmann J. An improved technique of eye drop self-administration for patients with limited vision. Am J Ophthalmol. 2003;135(4):530-533.

5. Sleath B, Robin AL, Covert D, Byrd JE, Tudor G, Svarstad B. Patientreported behavior and problems in using glaucoma medications. Ophthalmology. 2006;113(3):431-436.

6. Kholdebarin R, Campbell RJ, Jin Y, Buys YM. Multicenter study of compliance and drop administration in glaucoma. Can J Ophthalmol. 2008;43(4):454-461.

7. Taylor SA, Galbraith SM, Mills RP. Causes of non-compliance with drug regimens in glaucoma patients: a qualitative study. J Ocul Pharmacol Ther. 2002;18(5):401-409.

8. Krilis M, Coroneo M. Digital eye drop instillation. Aust Fam Physician. 2013;42(4):201-202.

9. Solomon A, Chowers I, Raiskup F, Siganos CS, Frucht-Pery J. Inadvertent conjunctival trauma related to contact with drug container tips: a masquerade syndrome. Ophthalmology. 2003;110:796-800.

10. Nelson J. Corneal abrasion resulting from a unit dose artificial tear dispenser. Am J Ophthalmol. 1987;103:333-334.

11. Aptel F, Masset H, Burillon C, Robin A, Denis P. The influence of disease severity on quality of eye-drop administration in patients with glaucoma or ocular hypertension. Br J Ophthalmol. 2009;93:700-701. 
12. Tsai T, Robin AL, Smith JP III. An evaluation of how glaucoma patients use topical medications: a pilot study. Trans Am Ophthalmol Soc. 2007;105: 29-35.

13. Kass MA, Hodapp E, Gordon M, Kolker AE, Goldberg I. Part I. Patient administration of eyedrops: interview. Ann Ophthalmol. 1982;14: 775-779.

14. McVeigh KA, Vakros G. The eye drop chart: a pilot study for improving administration of and compliance with topical treatments in glaucoma patients. Clin Ophthalmol. 2015;9:813-819.

15. Van Santvliet L, Ludwig A. Determinants of eye drop size. Surv Ophthalmol. 2004;49(2):197-213.

16. Gupta R, Patil B, Shah BM, Bali SJ, Mishra SK, Dada T. Evaluating eye drop instillation technique in glaucoma patients. J Glaucoma. 2012; 21(3):189-192.
17. Stone JL, Robin AL, Novack GD, Covert DW, Cagle GD. An objective evaluation of eyedrop instillation in patients with glaucoma. Arch Ophthalmol. 2009;127(6):732-736.

18. Centers for Medicare and Medicaid Services. Medicaid National Average Drug Acquisition Cost as of 2016-03-02. Baltimore, MD; 2016. Available from: https://data.medicaid.gov/Drug-Prices/NADAC-as-of2016-03-02/e9ia-h34v. Accessed July 01, 2016.

19. Geyer O, Bottone EJ, Podos SM, Schumer RA, Asbell PA. Microbial contamination of medications used to treat glaucoma. Br J Ophthalmol. 1995;79:376-379.
Clinical Ophthalmology

\section{Publish your work in this journal}

Clinical Ophthalmology is an international, peer-reviewed journal covering all subspecialties within ophthalmology. Key topics include: Optometry; Visual science; Pharmacology and drug therapy in eye diseases; Basic Sciences; Primary and Secondary eye care; Patien Safety and Quality of Care Improvements. This journal is indexed on

Submit your manuscript here: http://www.dovepress.com/clinical-ophthalmology-journal

\section{Dovepress}

PubMed Central and CAS, and is the official journal of The Society of Clinical Ophthalmology (SCO). The manuscript management system is completely online and includes a very quick and fair peer-review system, which is all easy to use. Visit http://www.dovepress.com/ testimonials.php to read real quotes from published authors. 